\title{
Inefficient Noncooperation in Networking Games of Common-Pool Resources
}

\author{
Hisao Kameda, Fellow, IEEE, and Eitan Altman, Senior Member, IEEE
}

\begin{abstract}
We study in this paper a noncooperative approach for sharing resources of a common pool among users, wherein each user strives to maximize its own utility. The optimality notion is then a Nash equilibrium. First, we present a general framework of systems wherein a Nash equilibrium is Pareto inefficient, which are similar to the 'tragedy of the commons' in economics. As examples that fit in the above framework, we consider noncooperative flow-control problems in communication networks where each user decides its throughput to optimize its own utility. As such a utility, we first consider the power which is defined as the throughput divided by the expected end-to-end packet delay, and then consider another utility of additive costs. For both utilities, we establish the non-efficiency of the Nash equilibria.
\end{abstract}

Index Terms-Braess paradox, common-pool resource, communication networks, flow control, Nash equilibrium, noncooperative game, Pareto inefficiency, power criterion, tragedy of the commons.

\section{INTRODUCTION}

There exist many systems where multiple independent users, or players, may strive to optimize each own utility unilaterally, which can be modeled as noncooperative games. As examples of the noncooperative games, communication networks like the Internet are joined by a number of independent users or organizations, like Internet service providers, that make decisions independently. Given users' decisions, the utilities of all users are determined. We call a situation where the decisions of all users are determined an allocation. The allocation where each user attains its own optimum coincidently is a Nash equilibrium. It is natural that these independent users seek their own benefits or utilities noncooperatively. Thus, such systems are regarded as noncooperative games.

Nash equilibria may be Pareto inefficient (or, simply, inefficient), that is, there may exist another allocation of a system where no users have less benefits and some have more benefits than in the Nash equilibrium of the system. In particular, we call an allocation of a system strongly Pareto inefficient if all users have more benefits in another allocation. Dubey [1] has shown that Nash equilibria may generally be Pareto inefficient based on the difference between the conditions to be satisfied by Nash equilibria and those to be satisfied by Pareto optima.

Manuscript received on August 7, 2007; revised March 8, 2008. The work of the first author is supported in part by the Grant-in-Aid for Scientific Research of Japan Society for the Promotion of Science. The work of the second author was supported in part by the BIONETs European Contract.

H. Kameda is with the Department of Computer Science, University of Tsukuba, Tsukuba Science City, Ibaraki 305-8573, Japan (e-mail: kameda@cs.tsukuba.ac.jp).

E. Altman is with INRIA Sophia Antipolis, B.P. 93, 06902 Sophia Antipolis Cedex, France (e-mail: Eitan.Altman@sophia.inria.fr).
It appears, however, to be difficult to obtain concrete cases of inefficient Nash equilibria from his result.

For communication and transportation networks, examples of such strong Pareto inefficiency have been shown with respect to noncooperative routing, first by Braess [2], and a number of related studies followed [3]-[10]. As for the noncooperative load balancing in distributed computer systems, the existence of paradoxes that are similar to that of Braess but that appear only in the case of a finite number of (atomic) players and not in the case of infinitesimal (nonatomic) players, in the same environment, has been shown [11], [12].

It is natural to think of noncooperative flow control and of the Nash equilibrium concept therein. It appears, however, that few studies have addressed the issue of Pareto inefficiency of Nash equilibria in noncooperative flow control. This article examines mainly this issue as examples. In addition, we note that the Nash equilibrium concept has been discussed with respect to the power control in wireless communications [13][15]. These kinds of problems are regarded as those in which players compete for common-pool resources. The examples of such problems as considered here have been studied in social science under the name, 'Tragedy of the Commons' (see, Hardin [16], Roemer [17], Roemer and Silvester [18], Funaki and Yamamoto [19], etc.).

This article first shows a fairly general framework of strongly Pareto-inefficient Nash equilibria. The framework may cover many examples of noncooperative games including noncooperative flow-control problems, noncooperative powercontrol problems in wireless networks, and a general problem named, the 'tragedy of commons' in social science as mentioned above. Therefore, the framework characterizes a class of noncooperative games that may be spread over various fields but may have a mutually similar structure.

As an example of the general framework, this article considers flow-control problems for communication networks with multiple ports of entry and of exit, where each user decides its throughput, that is, the rate of its packets to inject into a network so as to optimize its own performance objective unilaterally. As such an objective, we firstly consider the power that is defined as the throughput divided by the expected delay (the expected delay is the expected time for a packet to pass through the network) [20]. This unilaterally optimized allocation is a Nash equilibrium, the existence of which is proved here. We show that the Nash equilibrium is always strongly Pareto inefficient, and we identify an allocation that is Pareto superior to it. (We note that Korilis and Lazar showed the existence of Nash equilibria for networks consisting of state-dependent servers with one pair of ports of entry and exit where each user optimizes noncooperatively its throughput 
within its response time constraint; they did not mention inefficiency [21]. We note, in passing, that Mazumdar et al. [22] discussed cooperative flow control with the power criterion for optimization; they considered the Nash arbitration scheme in Jackson networks extensively, but mentioned briefly the Pareto inefficiency of the Nash equilibrium without showing its existence.)

Furthermore, for a subcategory of the noncooperative networks, we show that the degree of superiority [23] of a Pareto optimum to the Nash equilibrium increases as the number of users increases. Moreover, we present another flow-control setting with additive costs (instead of the power criterion) as another example of the general framework of strongly Paretoinefficient Nash equilibria.

\section{Organization of this paper}

The rest of this paper is organized as follows. Section II discusses a general framework of strongly Pareto-inefficient Nash equilibria. Section III discusses flow-control problems as examples of the properties shown in the above section. Subsections III-B, and also III-C, show that the Nash equilibrium of noncooperative flow-control on the network considered is always strongly Pareto inefficient. In Subsection III-B, we present inefficiency results on Nash equilibria for the flow control with power criterion. Subsection III-B1 shows more detailed estimates on the inefficient Nash equilibrium for a subcategory of the networks. Subsection III-B2 presents a simple example of the subcategory of the networks. In Subsection III-C, we present inefficiency results for the flow control with additive costs. Section IV concludes this article. The Appendix A presents a proof of the existence of a Nash equilibrium for each noncooperative flow control presented in Subsections III-B and III-C.

\section{A General Framework of Pareto-inefficient Nash EQUILIBRIA}

Consider a noncooperative game that has $n$ players where every player $i(i=1,2, \ldots, n)$ decides the value of $\lambda_{i} \geq 0$, that is, the strategy space of every player consists of nonnegative real numbers. Denote the set $\{1,2, \ldots, n\}$ by $\boldsymbol{n}$. Thus, the strategy profile is presented by a vector, $\lambda=\left(\lambda_{1}, \lambda_{2}, \ldots, \lambda_{n}\right)$. Let $U_{i}(\lambda)$ denote the utility that player $i$ strives to maximize. Let $\boldsymbol{L}$ be the product of the strategy spaces, that is, $\boldsymbol{L}=\left\{\lambda \mid \lambda_{i} \geq 0, i \in \boldsymbol{n}\right\}$. Denote by $\boldsymbol{C}(\subset \boldsymbol{L})$ the set of feasible values of $\lambda$. The definition of feasibility may depend on the system concerned. For example, for a stochastic system, such $\lambda$ for which the system is stable (for example, has a unique stationary regime) is feasible. $\boldsymbol{C}$ may have boundaries.

[Nash equilibrium] A strategy profile $\tilde{\lambda} \in \boldsymbol{C}$ is a Nash equilibrium (NE) if no unilateral deviation in strategy by any single player is profitable, that is, for all $i \in \boldsymbol{n}$ and for any $\lambda_{i}$ s.t. $\left(\lambda_{i}, \tilde{\lambda}_{(-i)}\right) \in \boldsymbol{C}, U_{i}\left(\tilde{\lambda}_{i}, \tilde{\lambda}_{(-i)}\right) \geq U_{i}\left(\lambda_{i}, \tilde{\lambda}_{(-i)}\right)$, where $\lambda_{(-i)}$ denotes the (n-1)-vector resulting from $\lambda$ with the $i$ th element $\lambda_{i}$ being removed. We assume that a Nash equilibrium exists in $\boldsymbol{C}$.

[Pareto inefficiency] In the case where $\forall i, U_{i}(\bar{\lambda}) \geq U_{i}(\hat{\lambda})$ and $\exists i, U_{i}(\bar{\lambda})>U_{i}(\hat{\lambda}), \bar{\lambda}$ is Pareto superior to $\hat{\lambda}$ and $\hat{\lambda}$ is Pareto inferior to $\bar{\lambda}$. In the case where there exists $\lambda \in C$ s.t. $\lambda$ is
Pareto superior to $\hat{\lambda}, \hat{\lambda}$ is Pareto inefficient. In the case where $\forall i, U_{i}(\bar{\lambda})>U_{i}(\hat{\lambda})$, we say that $\bar{\lambda}$ is strongly Pareto superior to $\hat{\lambda}$ and that $\hat{\lambda}$ is strongly Pareto inferior to $\bar{\lambda}$. In the case where there exists $\lambda \in \boldsymbol{C}$ s.t. $\lambda$ is strongly Pareto superior to $\hat{\lambda}$, we say that $\hat{\lambda}$ is strongly Pareto inefficient.

Let $\tilde{\lambda}(\in \boldsymbol{C})$ denote a strategy profile that presents a Nash equilibrium. Denote the set $\left\{i \mid \tilde{\lambda}_{i}\right.$ is not a boundary value of $\boldsymbol{C}$ by $\tilde{\boldsymbol{n}}$. Denote a neighborhood of $\lambda$ by $V(\boldsymbol{\lambda})(\subset \boldsymbol{C})$. We introduce the following assumptions on a Nash equilibrium $\tilde{\lambda}$ :

Assumption $\Psi 1$. For a Nash equilibrium $\tilde{\lambda}$, the partial derivatives of $U_{i}(\lambda)$ for all $i \in \boldsymbol{n}$ with respect to all $\lambda_{j}, j \in \tilde{\boldsymbol{n}}$, exist and are continuous in $\lambda \in V(\tilde{\lambda})$, and either of the following two cases holds:

1) For all $i \in \boldsymbol{n}$, the utility, $U_{i}$, of player $i$ is decreasing in $\lambda_{j}$, for all $j \in \tilde{\boldsymbol{n}}, j \neq i$, that is,

$$
\left.\frac{\partial U_{i}}{\partial \lambda_{j}}\right|_{\lambda=\tilde{\lambda}}<0, \text { for all } j \in \tilde{\boldsymbol{n}}(j \neq i) .
$$

2) For all $i \in \boldsymbol{n}$, the utility, $U_{i}$, of player $i$ is increasing in $\lambda_{j}$, for all $j \in \tilde{\boldsymbol{n}}, j \neq i$, that is,

$$
\left.\frac{\partial U_{i}}{\partial \lambda_{j}}\right|_{\lambda=\tilde{\lambda}}>0, \text { for all } j \in \tilde{\boldsymbol{n}}(j \neq i) .
$$

Assumption $\Psi 2$. For a Nash equilibrium $\tilde{\lambda}$, more than one element of $\tilde{\lambda}$ is not a boundary value, but is an interior value. Then, $\tilde{\boldsymbol{n}}$ has more than one element, i.e., $|\tilde{\boldsymbol{n}}| \geq 2$. That is, the strategies $\tilde{\lambda}_{i}$ of at least two users are of interior values.

From the definition of $\tilde{\boldsymbol{n}}$,

$$
\left.\frac{\partial U_{i}}{\partial \lambda_{i}}\right|_{\lambda=\tilde{\lambda}}=0 \text { for } i \in \tilde{\boldsymbol{n}} .
$$

Theorem 1: If Assumptions $\Psi 1$ and $\Psi 2$ hold for a Nash equilibrium in $\boldsymbol{C}$, it is strongly Pareto inefficient.

Proof: Consider reducing the values of all elements $\lambda_{i}$, $i \in \tilde{\boldsymbol{n}}$, of $\lambda$ from $\tilde{\lambda}_{i}$ coincidently. Let $\delta>0$ be a small positive value. Let $\delta_{i}=\delta$ for $i \in \tilde{\boldsymbol{n}}$ and $\delta_{i}=0$ for $i \notin \tilde{\boldsymbol{n}}$. Denote the vector $\left(\tilde{\lambda}_{1}-\delta_{1}, \tilde{\lambda}_{2}-\delta_{2}, \ldots, \tilde{\lambda}_{n}-\delta_{n}\right)$ by $\tilde{\lambda}-\boldsymbol{\delta}$.

Then, we have for $i \in \boldsymbol{n}$,

$$
\begin{gathered}
U_{i}(\tilde{\boldsymbol{\lambda}}-\boldsymbol{\delta})=U_{i}(\tilde{\lambda})-\left.\frac{\partial U_{i}}{\partial \lambda_{i}}\right|_{\lambda=\tilde{\lambda}} \delta-\left.\sum_{q \neq i, q \in \tilde{\boldsymbol{n}}} \frac{\partial U_{i}}{\partial \lambda_{q}}\right|_{\lambda=\tilde{\lambda}} \delta+o(\delta), i \in \tilde{\boldsymbol{n}}, \\
U_{i}(\tilde{\boldsymbol{\lambda}}-\boldsymbol{\delta})=U_{i}(\tilde{\lambda})-\left.\sum_{q \in \tilde{n}} \frac{\partial U_{i}}{\partial \lambda_{q}}\right|_{\lambda=\tilde{\lambda}} \delta+o(\delta), \quad i \notin \tilde{\boldsymbol{n}} .
\end{gathered}
$$

by noting that $\delta_{j}=0, j \notin \tilde{\boldsymbol{n}}$ (since $\tilde{\lambda}_{j}, j \notin \tilde{\boldsymbol{n}}$, is on a boundary). Note that $o(\delta) / \delta \rightarrow 0$ as $\delta \rightarrow 0$.

Consider the case 1) of Assumption $\Psi 1$. Note, in the case of eq. (2), for all $i \in \tilde{\boldsymbol{n}}$, that $\left.\frac{\partial U_{i}}{\partial \lambda_{i}}\right|_{\lambda=\tilde{\lambda}}=0$ (since $\tilde{\lambda}$ is a Nash equilibrium) by Assumption $\Psi 2$ (eq. (1)). Note, furthermore, in the cases of eqs. (2) and (3), that the coefficient $\left.\frac{\partial U_{i}}{\partial \lambda_{q}}\right|_{\lambda=\tilde{\lambda}}<0$ for all $q \in \tilde{\boldsymbol{n}}(q \neq i)$ by Assumption $\Psi 1$. Therefore, $U_{i}(\tilde{\boldsymbol{\lambda}}-\boldsymbol{\delta})>$ $U_{i}(\tilde{\lambda})$ for all $i$, since $\delta_{i}=\delta>0$ for all $i \in \tilde{\boldsymbol{n}}$. This implies that there exists a value of $\lambda(=\tilde{\lambda}-\delta \neq \tilde{\lambda})$ such that $U_{i}(\lambda)>U_{i}(\tilde{\lambda})$ for all $i \in \boldsymbol{n}$. 
Similarly for the case 2) of Assumption $\Psi 1$.

Remark 1: We note that even user $i$ who has a boundary value of $\tilde{\lambda}_{i}$ can increase its utility $U_{i}$ due to the change of $\lambda_{i}$, $i \in \tilde{\boldsymbol{n}}$.

Note furthermore the case where $\tilde{\boldsymbol{n}}$ has only one element $i$ (that is, Assumption $\Psi 2$ is violated). Then, from (2), $U_{i}(\tilde{\lambda}-\delta)=U_{i}(\tilde{\lambda})+o(\delta)$ for $i \in \tilde{\boldsymbol{n}}$, and we cannot simply see whether $U_{i}$ increases or decreases as $\lambda$ moves from $\tilde{\lambda}$ (only by investigating the first-order derivatives). We thus see that Assumption $\Psi 2(|\tilde{\boldsymbol{n}}| \geq 2)$ supports Theorem 1 in a simple way.

Remark 2: Here we mention a few among vastly many examples that fit in the general framework given in this section. Alpcan et al. [24] showed the existence of a Nash equilibrium for their system. Similar models of the power control in wireless communications can be found [13], [15], [25], [26]. Then, we may apply Theorem 1 to these examples, and may show that a Nash equilibrium of them is Pareto inefficient.

As some concrete examples that fit in the general framework mentioned above, in the following section III, we present some results on flow control in BCMP-type networks [27], where the utility of each use is (1) power criterion (Subsection III-B) in some details or (2) criterion based on additive costs (Subsection III-C) briefly.

\section{Flow Control in Networks}

We examine some flow control problems in networks that fit in the above mentioned general framework given in Section II.

\section{A. Assumptions on Networks}

Consider a communication network modeled by an open product-form network of $m$ state-independent queues, $k=$ $1,2, \ldots, m$ (that model communication links, or, simply, links) [27]. Denote the set $\{1,2, \ldots, m\}$ by $\boldsymbol{m}$. The vertices or nodes connected by links model the routers of the communication network. There are $n$ independent users, $1,2, \ldots, n$ as before. User $i$ decides the rate $\lambda_{i}$ of packets to pass through a communication network so that the utility, $U_{i}$, of the user $i$ may be maximal. Denote the vector $\left(\lambda_{1}, \lambda_{2}, \ldots, \lambda_{n}\right)$ by $\lambda . T_{i}$ is the expected end-to-end delay of the packets in control of user $i$. Note, in the following, that the domain of index variables $i, j$, and $r$ is $\boldsymbol{n}$ and that of $l$ and $k$ is $\boldsymbol{m}$.

$\mu_{i k}$ is the state-independent service rate of user- $i$ packets at link $k$. In this article, it is assumed that each router (or, node) has a sufficient capacity of storing packets, and, thus, losses of packets may not occur. $q_{i k}$ is the resulting visit rate of user- $i$ packets to link $k$. That is, $q_{i k}$, for all $i, k$, is the solution of the following system of equations:

$$
q_{i k}=p_{0 k}^{i}+\sum_{l} q_{i l} p_{l k}^{i} \text { for all } i, k,
$$

where $p_{l k}^{i}$ and $p_{0 k}^{i}$, respectively, are the probabilities that a user- $i$ packet goes to link $k$ after leaving link $l$ and when it enters the network, and are fixed and not subject to optimal control (we are concerned only with optimal flow control and not with optimal routing in this paper). Then, if user $i$ injects the rate $\lambda_{i}$ of packets into the network, user- $i$ packets visit link $k$ at the rate of $q_{i k} \lambda_{i}$. User $i$ injects the rate, $p_{0 k}^{i} \lambda_{i}$, of packets into link $k$ from the outside of the network. User- $i$ packets departing from link $k$ leave the network at the frequency (or, probability) $q_{k 0}^{i}$. That is, the network has multiple ports of entry and of exit. Consider the case where the mean response time, $T_{i}^{(k)}$, for a user- $i$ packet to pass through link $k$, is

$$
\begin{gathered}
T_{i}^{(k)}=\mu_{i k}^{-1} T^{(k)} \text { and } T^{(k)}=\frac{1}{1-s_{l} \sum_{r} q_{r k} \lambda_{r} / \mu_{r k}}, \\
\text { if } 1-s_{l} \sum_{r} q_{r k} \lambda_{r} / \mu_{r k}>0, \text { otherwise infinite, }
\end{gathered}
$$

where $s_{l}$ is 1 for a link modeled by a single-server, $1 / h$ for a link consisting of $h$ parallel channels each of which is chosen with probability $1 / h$ and is modeled by a single server, and 0 for a link modeled by an infinite server, for $1-s_{l} \sum_{r} q_{r k} \lambda_{r} / \mu_{r k}>$ 0 [27]. Denote $\boldsymbol{k}=\left\{l \mid s_{l} \neq 0\right\}$. Then, using the Little's result,

$$
\begin{gathered}
T_{i}(\lambda)=\sum_{l \in \boldsymbol{k}} \frac{Q_{i l}}{1-s_{l} \sum_{r} Q_{r l} \lambda_{r}}+\sum_{l \in \boldsymbol{m}-\boldsymbol{k}} Q_{i l}, \\
\text { if } 1-s_{l} \sum_{r} Q_{r l} \lambda_{r}>0 \text { for all } l, \text { otherwise infinite, } \\
\text { where } Q_{i l}=\frac{q_{i l}}{\mu_{i l}} .
\end{gathered}
$$

Clearly, $T_{i}(\lambda)$ is increasing in $\lambda$. We note that $\sum_{l \in m-k} Q_{i l}$ is constant and independent of the strategy. In order that the statistical equilibrium of this network be attained, it must hold that $\lambda \in \boldsymbol{C}$, where the feasible region $\boldsymbol{C}$ is

$$
\boldsymbol{C}=\left(\lambda \mid \lambda_{i} \geq 0, i \in \boldsymbol{n}, \text { and } 1-s_{l} \sum_{r} Q_{r l} \lambda_{r}>0, l \in \boldsymbol{k}\right) .
$$

Furthermore, define regions $\overline{\boldsymbol{C}}$ and $\hat{\boldsymbol{C}}$ such that

$$
\begin{aligned}
& \overline{\boldsymbol{C}}=\left(\lambda \mid \lambda_{i}>0, i \in \boldsymbol{n}, \text { and } 1-s_{l} \sum_{r} Q_{p l} \lambda_{r}>0, l \in \boldsymbol{k}\right), \\
& \hat{\boldsymbol{C}}=\left(\lambda \mid \lambda_{i} \geq 0, i \in \boldsymbol{n}, \text { and } 1-s_{l} \sum_{r} Q_{r l} \lambda_{r} \geq 0, l \in \boldsymbol{k}\right) .
\end{aligned}
$$

Note that $\hat{\boldsymbol{C}}$ is a closed and bounded subset of $\lambda . \hat{\boldsymbol{C}}-\overline{\boldsymbol{C}}$ comprises the boundary consisting of $n+k$ hyperplanes each with $(n-1)$-dimensions, $n$ from $\lambda_{i}=0, i \in \boldsymbol{n}$, and $k$ from $1-s_{l} \sum_{r} Q_{r l} \lambda_{r}=0, l \in \boldsymbol{k}$. We call the part of the boundary consisting of $\lambda_{i}=0$, the $(i-0)$ policy boundary, and the part of boundary which is not any of $(i-0)$ policy boundary, $i \in \boldsymbol{n}$, the capacity boundary. We also define for convenience,

$$
A_{i l}=1-s_{l} \sum_{r \neq i} Q_{r l} \lambda_{r} .
$$

a) Utility of each user: Each of network users (user-i) has two important major concerns in choosing the protocol to use: one is the amount of packets user- $i$ can send per unit time (throughput), denoted by $\lambda_{i}$, and the other is the expected time of each packet taken from its origin to its destination (mean response time), denoted by $T_{i}$. As the utility of each user- $i$, we need to consider one scalar value taking account of the above both $\lambda_{i}$ and $T_{i}$. That is, in general we are interested in criteria that will allow us to represent preference to high throughput and to low delay. Both the additive criterion as well 
as the power criterion fall into this category. More generally, since the Nash equilibrium is unchanged if we replace the utility by the logarithm of the utility, the power criterion can be transformed (using the logarithm) into an additive criterion: the logarithm utility is the sum of the difference between the $\log$ of the throughput and the log of the delay.

In this paper, as such utilities as above, we examine, in particular, the power criterion as in Subsection III-B and the criterion based on some additive costs as in Subsection III-C. We cannot afford to go into the detailed discussion of protocols (see, for example, [28]).

\section{B. Noncooperative Flow Control with Power Criterion}

The simplest reasonable utility of user- $i$ that consists of $\lambda_{i}$ and $T_{i}$ looks to be the power criterion as defined as follows: The power is defined as $P_{i}=\lambda_{i} / T_{i}$ for user- $i$. The power criterion has been widely used [20]-[22], [29]-[35]. Although we found some criticism of this criterion in [36], we also found many arguments for using it [32], [35]. We shall add to it another interpretation of this criterion as follows. Assume that each flow that is controlled by one user is the aggregation of several mini flows, some belonging to applications that wish to minimize the delay (that is, maximize the inverse of the delay) as their utilities and others that wish to maximize the throughput as their utilities. Suppose that we wish to have one utility of the user that would represent some fair tradeoff between the two distinct utilities. Then, if we adopt a concept that embodies the spirit of the proportional fairness concept (or equivalently, the Nash bargaining solution concept (see, for example, [37])) for the fair tradeoff among the utilities with respect to the user, then the utility of the user we obtain is precisely the power.

In this subsection, we consider the case where the utility, $U_{i}$, of user $i$ is its power, $P_{i}$, i.e., $U_{i}=P_{i}$ for all $i$. Denote the vector $\left(P_{1}, P_{2}, \ldots, P_{n}\right)$ by $\boldsymbol{P}$. From $(6), P_{i}(\lambda)$ is defined for all $\lambda \in \boldsymbol{L}$, and $P_{i}(\lambda)=0$ for $\lambda \in \boldsymbol{L}-\overline{\boldsymbol{C}}$ and $i \in \boldsymbol{n}$. From (6) and the definition $P_{i}=\lambda_{i} / T_{i}$, we see that $\boldsymbol{P}(\lambda)$ is continuous in $\lambda$. By noting that, for $\lambda \in \boldsymbol{L}-\hat{\boldsymbol{C}}, P_{i}(\lambda)=0$ for all $i$, the set $\boldsymbol{\pi}$ of all possible values of $\boldsymbol{P}(\lambda)$ is given by $\lambda \in \hat{\boldsymbol{C}}$. Since $\hat{\boldsymbol{C}}$ is closed and bounded and $\boldsymbol{P}(\boldsymbol{\lambda})$ is continuous in $\lambda, \boldsymbol{\pi}$ is also closed and bounded. The existence of a Nash equilibrium flow control will be shown later by Theorem 2 .

b) Power maximization by each user:

Lemma 1: Given $\lambda_{j}$, for all $j \neq i$, the value $\check{\lambda}_{i}$ of $\lambda_{i}$ that maximizes $P_{i}(\lambda)$ is uniquely given for all $i$ such that

$$
\left.0<\check{\lambda}_{i}<\min _{l}\left\{A_{i l} /\left(s_{l} Q_{i l}\right)\right\}\right\} .
$$

Proof: From (6), for $\lambda_{i}>0$ and $1-s_{l} \sum_{r} Q_{r l} \lambda_{r}=$ $1-A_{i l}-s_{l} Q_{i l} \lambda_{i}>0$ for all $l$, we have the following, for $i \in \boldsymbol{n}$ :

$$
\begin{aligned}
& P_{i}^{-1}(\lambda)=\frac{T_{i}(\lambda)}{\lambda_{i}}=\sum_{l \in \boldsymbol{k}} \frac{Q_{i l}}{\lambda_{i}\left(1-s_{l} \sum_{r} Q_{r l} \lambda_{r}\right)}+\frac{1}{\lambda_{i}} \sum_{l \in \boldsymbol{m}-\boldsymbol{k}} Q_{i l} \\
& =\sum_{l \in \boldsymbol{k}}\left(\frac{1}{\lambda_{i}}+\frac{s_{l} Q_{i l}}{1-s_{l} \sum_{r} Q_{r l} \lambda_{r}}\right) \frac{Q_{i l}}{1-s_{l} \sum_{r \neq i} Q_{r l} \lambda_{r}}+\frac{1}{\lambda_{i}} \sum_{l \in \boldsymbol{m}-\boldsymbol{k}} Q_{i l} .
\end{aligned}
$$

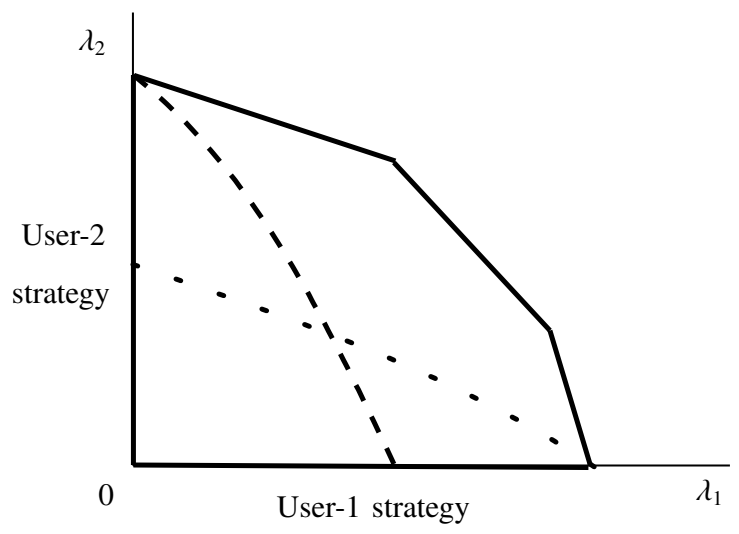

Fig. 1. A Nash equilibrium allocation

The partial differential coefficients of $P_{i}^{-1}$ are the following, for $i \in \boldsymbol{n}$ :

$$
\begin{gathered}
\frac{\partial}{\partial \lambda_{i}} P_{i}^{-1}=-\frac{1}{\lambda_{i}^{2}}\left(\sum_{l \in \boldsymbol{k}} \frac{Q_{i l}}{1-s_{l} \sum_{r \neq i} Q_{r l} \lambda_{r}}+\sum_{l \in \boldsymbol{m}-\boldsymbol{k}} Q_{i l}\right) \\
+\sum_{l \in \boldsymbol{k}} \frac{s_{l}^{2} Q_{i l}^{3}}{\left(1-s_{l} \sum_{r \neq i} Q_{r l} \lambda_{r}\right)\left(1-s_{l} \sum_{r} Q_{r l} \lambda_{r}\right)^{2}} \\
=-\frac{1}{\lambda_{i}^{2}}\left(\sum_{l \in \boldsymbol{k}} \frac{Q_{i l}}{A_{i l}}+\sum_{l \in \boldsymbol{m}-\boldsymbol{k}} Q_{i l}\right)+\sum_{l \in \boldsymbol{k}} \frac{s_{l}^{2} Q_{i l}^{3}}{A_{i l}\left(A_{i l}-s_{l} Q_{i l} \lambda_{i}\right)^{2}} .
\end{gathered}
$$

(14) is derived from (13). Note that $A_{i l}$ and $Q_{i l}$ are independent of $\lambda_{i}$, as seen from rel. (6) and def. (10). Also note that, for sufficiently small $\lambda_{i}>0$, the extreme right-hand side of (14) is negative whereas for sufficiently large $\lambda_{i}$ s.t. $A_{i l}-s_{l} Q_{i l} \lambda_{i}>0$, $l \in \boldsymbol{k}$, it is positive. Furthermore, note that the $\lambda_{i}$ 's in the extreme right-hand side of eq. (14) are in the denominators and have negative signs in front of them. Then, it can be easily seen that, since both the first and second terms of the extreme righthand side of (14) are increasing in $\lambda_{i}$ for $0<\lambda_{i}<A_{i l} / s_{l} Q_{i l}$, $l \in \boldsymbol{k}$, given $\lambda_{j}$, for all $j \neq i$, there is a unique value, $\breve{\lambda}_{i}$, of $\lambda_{i}$ that makes (14) to be zero and, thus, maximizes the power $P_{i}$, given $\lambda_{j}$ for all $j \neq i$. That is, from (14), for $\check{\lambda}_{i}$ such that (11) holds,

$$
\sum_{l \in \boldsymbol{k}} \frac{Q_{i l}}{A_{i l}}+\sum_{l \in \boldsymbol{m}-\boldsymbol{k}} Q_{i l}=\sum_{l \in \boldsymbol{k}} \frac{Q_{i l}}{A_{i l}}\left(\frac{A_{i l}}{s_{l} Q_{i l} \check{\lambda}_{i}}-1\right)^{-2},
$$

It is evident that such a value of $\check{\lambda}_{i}$ is unique and satisfies the conditions that $\check{\lambda}_{i}>0$ and $A_{i l}-s_{l} Q_{i l} \check{\lambda}_{i}=1-s_{l} \sum_{r \neq i} Q_{r l} \lambda_{r}-$ $s_{l} Q_{i l} \breve{\lambda}_{i}>0$ for all $l$. Since, for such $\lambda_{i}$ as does not satisfy the conditions, from (6) and from the definition of the power of user- $\left.i\left(P_{i}=\lambda_{i} / T_{i}\right)\right), P_{i}=0$, then such $\lambda_{i}$ as does not satisfy the conditions will not be chosen by any user- $i$.

c) Nash equlibrium of the power flow control: Denote by $\lambda^{(-i)}$ an $(n-1)$-dimensional vector that has elements, $\lambda_{r}, r \in \boldsymbol{n}-\{i\}$, of $\lambda$. Denote by $\check{\lambda}_{i}\left(\lambda^{(-i)}\right)$ the value of $\lambda_{i}$ that maximizes $P_{i}$ given the values of the other $\lambda_{j}$ for all $j \neq i$. Clearly, the value of $\tilde{\lambda}=\left(\tilde{\lambda}_{1}, \tilde{\lambda}_{2}, \ldots, \tilde{\lambda}_{n}\right)$ that satisfies $\tilde{\lambda}_{i}=\breve{\lambda}_{i}\left(\tilde{\lambda}^{(-i)}\right)$ for all $i$ is a Nash equilibrium. From (15), we see that $\tilde{\lambda}$ is not on any boundary of $\boldsymbol{C}$. That is, $\tilde{\boldsymbol{n}}=\boldsymbol{n}$ where $\tilde{\boldsymbol{n}}$ is defined before Assumption $\Psi 1$. Denote by $\mathbf{0}$ a vector for which all the elements have the value, zero. We can see that $\left\{\left(\check{\lambda}_{i}\left(\lambda^{(-i)}\right), \lambda^{(-i)}\right) \mid \lambda \in C\right\}$, comprises an $(n-1)$-dimensional 
hypersurface that connects the allocation $\left(\check{\lambda}\left(\mathbf{0}^{(-i)}\right), \mathbf{0}^{(-i)}\right)$ and $(n-2)$-dimensional hypersurface that is the intersection of the $(i-0)$ policy boundary and the capacity boundary. In total, there exist $n$ of these $(n-1)$-dimensional hypersurfaces, one for each $i(\in \boldsymbol{n})$, and the intersection of all these hypersurfaces will be one (and possibly only one) allocation, which is a Nash equilibrium. Fig. 1 illustrates a case where $n=2$ and $k=3$. The solid lines show the boundary. The dashed curve consists of the allocations $\left(\check{\lambda}_{1}, \lambda_{2}\right)$ where each $\breve{\lambda}_{1}$ is the strategy optimal to user 1 , given the strategy of user $2, \lambda_{2}$. The dotted curve consists of the allocations $\left(\lambda_{1}, \breve{\lambda}_{2}\right)$ where each $\check{\lambda}_{2}$ is the strategy optimal to user 2 , given the strategy of user $1, \lambda_{1}$. The intersection of the dashed and dotted curves shows a Nash equilibrium allocation.

A proof of the existence of a Nash equilibrium, $\tilde{\lambda} \in \boldsymbol{C}$, can be shown based on the Kakutani fixed-point theorem [38].

Theorem 2: There exists a Nash equilibrium flow control solution, $\tilde{\lambda} \in \boldsymbol{C}$, for this network.

Proof: See Appendix A.

Corollary 1: Nash equilibria for flow control where each user optimizes its power are always strongly Pareto inefficient.

Proof: Note that, from (12), by noting $U_{i}=1 / P_{i}^{-1}$, we have

$$
\frac{\partial U_{i}}{\partial \lambda_{j}}=-\left(P_{i}\right)^{2} \frac{\partial P_{i}^{-1}}{\partial \lambda_{j}}=-\left(P_{i}\right)^{2} \sum_{l \in \boldsymbol{k}}\left\{\frac{s_{l} Q_{i l} Q_{q l}}{\lambda_{i}\left(1-s_{l} \sum_{r} Q_{r l} \lambda_{r}\right)^{2}}\right\}<0
$$

$$
i, j \in \boldsymbol{n}(j \neq i)
$$

Then, $\left.\frac{\partial U_{i}}{\partial \lambda_{j}}\right|_{\lambda=\tilde{\lambda}}<0$ for all $i, j \in \boldsymbol{n}(j \neq i)$. Note, furthermore, that $\left.\frac{\partial U_{i}}{\partial \lambda_{i}}\right|_{\lambda=\tilde{\lambda}}=0$ for all $i \in \boldsymbol{n}$ (since $\tilde{\lambda}$ is a Nash equilibrium). Thus, we see that Assumptions $\Psi 1$ [Case 1)] and $\Psi 2$ hold since $\tilde{\boldsymbol{n}}=\boldsymbol{n}$. Therefore, from Theorem 1 follows this property.

Remark 3: It is to be noted that the powers of all users improve by reducing the throughputs, $\lambda$, of all users from the Nash equilibrium coincidently (similarly as in the proof of Theorem 1).

1) More Concrete Results on the Flow Control in a Subcategory of the Networks: We consider a subcategory of the networks that satisfy the following assumption.

Assumption $\Pi 1$ Given $\lambda, T_{i}, i \in \boldsymbol{n}$, is given by a function $D(\rho)$ with a parameter $\mu_{i}(>0), i \in \boldsymbol{n}$, as follows:

$$
T_{i}(\lambda)=\frac{1}{\mu_{i}} D(\rho), \text { where } \rho=\sum_{r} \rho_{r}, \text { and } \rho_{i}=\frac{\lambda_{i}}{\mu_{i}} .
$$

$D(\rho)$, defined for $\rho \geq 0$, satisfies the following: $D(0)=\Delta$ (where $\Delta$ is a positive constant), $D(\rho)$ is increasing, and $\log D(\rho)$ is convex.

Assumption $\Pi 1$ is satisfied, for example, by the following network. Consider, in particular, the case where $q_{i k} / \mu_{i k}(=$ $\left.Q_{i k}\right)=\gamma_{k} / \mu_{i}$ for some $\gamma_{k}$, for all $i, k$. This can be satisfied, for example, when there exists $\delta_{i k}$ such that $\mu_{i k}=\delta_{i k} \mu_{i}$ and $q_{i k}=\delta_{i k} \gamma_{k}$, for all $i, k$. In this case, the ratio of the mean response time of a user- $i$ packet to pass through link $k$ to that of a user- $j$ packet is $\mu_{j} / \mu_{i}$ (the same for all $k$ ) for all $i, j, k$. For example, the service rate $\mu_{i}$ of each node may be distinct among users $i \in \boldsymbol{n}$ but is the same for all nodes $k \in \boldsymbol{m}$ with respect to each user $i$, whereas the visit rate $\gamma_{k}$ at each node may be distinct among nodes $k \in \boldsymbol{m}$ but is the same for all users $i \in \boldsymbol{n}$ with respect to each node.

Define $\rho_{i}=\lambda_{i} / \mu_{i}$ and $\rho=\sum_{r} \rho_{r}$. Then, from the relations (4), (5), and (6) on the networks given in Subsection III-A, we have

$$
T_{i}=\frac{1}{\mu_{i}} D(\rho), \quad D(\rho)=\sum_{l \in \boldsymbol{k}} \frac{\gamma_{l}}{1-s_{l} \gamma_{l} \rho}+\sum_{l \in \boldsymbol{m}-\boldsymbol{k}} \gamma_{l} .
$$

From the above, we have

Proposition 1: For $D(\rho)$ given by (18) and $0 \leq \rho<$ $\min _{l \in \boldsymbol{k}}\left\{1 /\left(s_{l} \gamma_{l}\right)\right\}, \log D(\rho)$ is increasing and convex in $\rho$.

Proof: From (18) and the following Lemma 2, follows this proposition.

Lemma 2: For $D(\rho)$ given by (18) and $0 \leq \rho<$ $\min _{k}\left\{1 /\left(s_{k} \gamma_{k}\right)\right\}, D(\rho) / D^{\prime}(\rho)$ is decreasing in $\rho$.

Proof: Note that

$$
D^{\prime}(\rho)=\sum_{l \in \boldsymbol{k}} s_{l}\left(\frac{\gamma_{l}}{1-s_{l} \gamma_{l} \rho}\right)^{2}
$$

Without losing generality, $l=1,2, \ldots, m$, can be renumbered such that $s_{1} \gamma_{1}=\min _{l \in k}\left\{s_{l} \gamma_{l}\right\}$. Then,

$$
\begin{aligned}
& D(\rho)=\sum_{l \in \boldsymbol{k}}\left(\frac{\gamma_{l}}{1-s_{l} \gamma_{l} \rho}\right)^{2} \frac{1-s_{l} \gamma_{l} \rho}{\gamma_{l}}+\sum_{l \in \boldsymbol{m}-\boldsymbol{k}} \gamma_{l} \\
&=\frac{1-s_{1} \gamma_{1} \rho}{s_{1} \gamma_{1}} \sum_{l \in \boldsymbol{k}} s_{l}\left(\frac{\gamma_{l}}{1-s_{l} \gamma_{l} \rho}\right)^{2} \\
&+\sum_{l \in \boldsymbol{k}, l \neq 1} s_{l}\left(\frac{\gamma_{l}}{1-s_{l} \gamma_{l} \rho}\right)^{2}\left(\frac{1}{s_{l} \gamma_{l}}-\frac{1}{s_{1} \gamma_{1}}\right)+\sum_{l \in \boldsymbol{m}-\boldsymbol{k}} \gamma_{l} .
\end{aligned}
$$

Therefore,

$$
\begin{gathered}
\frac{D(\rho)}{D^{\prime}(\rho)}=\frac{\sum_{l \in \boldsymbol{k}}\left(\frac{\gamma_{l}}{1-s_{l} \gamma_{l} \rho}\right)^{2} \frac{1-s_{l} \gamma_{l} \rho}{\gamma_{l}}+\sum_{l \in \boldsymbol{m}-\boldsymbol{k}} \gamma_{l}}{\sum_{l \in \boldsymbol{k}} s_{l}\left(\frac{\gamma_{l}}{1-s_{l} \gamma_{l} \rho}\right)^{2}} \\
=\frac{1-s_{1} \gamma_{1} \rho}{s_{1} \gamma_{1}}+\frac{\sum_{l \in \boldsymbol{k}, l \neq 1} s_{l}\left(\frac{\gamma_{l}}{1-s_{l} \gamma_{l} \rho}\right)^{2}\left(\frac{1}{s_{l} \gamma_{l}}-\frac{1}{s_{1} \gamma_{1}}\right)+\sum_{l \in \boldsymbol{m}-\boldsymbol{k}} \gamma_{l}}{\sum_{l} s_{l}\left(\frac{\gamma_{l}}{1-s_{l} \gamma_{l} \rho}\right)^{2}} .
\end{gathered}
$$

Thus, by noting that $s_{1} \gamma_{1} \leq s_{k} \gamma_{k}$ for all $k$, we see that $D(\rho) / D^{\prime}(\rho)$ is decreasing in $\rho$.

From Assumption П1,

$$
P_{i}=\frac{\lambda_{i}}{T_{i}}=\frac{\lambda_{i} \mu_{i}}{D(\rho)}=\frac{\mu_{i}^{2} \rho_{i}}{D(\rho)} .
$$


a) Noncooperative flow control: From (20)

$$
\frac{\partial}{\partial \rho_{i}}\left(\log P_{i}\right)=\frac{1}{\rho_{i}}-\frac{D^{\prime}(\rho)}{D(\rho)}, \text { for } \rho_{i}>0 .
$$

Thus, the set of the values $\tilde{\rho}_{i}$ of $\rho_{i}$ s.t. $\tilde{\rho}_{i}=D(\tilde{\rho}) / D^{\prime}(\tilde{\rho})$, for all $i$, presents a Nash equilibrium, where

$$
\tilde{\rho}=\frac{n D(\tilde{\rho})}{D^{\prime}(\tilde{\rho})} .
$$

Then, the noncooperative optimum flow for user $i$ is $\tilde{\lambda}_{i}=\mu_{i} \tilde{\rho}_{i}$.

b) Cooperative flow control: Consider an overall measure, $O=\sum_{i} \mu_{i}^{-2} P_{i}$. We note that the solution that optimizes the measure is Pareto optimal. It may not be so called social optimal. From (20),

$$
\begin{gathered}
O=\sum_{i} \mu_{i}^{-2} P_{i}=\frac{\rho}{D(\rho)}, \\
\frac{\partial}{\partial \rho}(\log O)=\frac{1}{\rho}-\frac{D^{\prime}(\rho)}{D(\rho)}, \text { for } \rho>0 .
\end{gathered}
$$

Then, an overall optimum for this overall measure $O$ is given by such a value $\hat{\rho}$ of $\rho$ that

$$
\hat{\rho}=\frac{D(\hat{\rho})}{D^{\prime}(\hat{\rho})} .
$$

There are distinct sets of flows for users that results in $\hat{\rho}$ and achieves this overall optimum. One set of flows for users that gives the above overall optimum is given by $\lambda_{i}=\mu_{i} \hat{\rho} / n$ and $\hat{\rho}_{i}=\hat{\rho} / n, i \in \boldsymbol{n}$. Thus, the set of flows for users $\lambda_{i}=\mu_{i} \hat{\rho} / n$ is a Pareto-optimal flow control.

c) Comparison of noncooperative vs. cooperative flow controls: Denote by $\tilde{P}_{i}$ and $\hat{P}_{i}$ the powers of user $i$ in the noncooperative and the above-mentioned overall optimal flowcontrol solutions, respectively. The following property holds in the setting of the model.

Theorem 3: There exists a unique Nash equilibrium of noncooperative flow control, and it is always strongly Pareto inferior to the overall optimum defined above, that is, for all $i, \tilde{P}_{i}<\hat{P}_{i}$. The power of each user in an overall optimum is proportionate to that in the Nash equilibrium, that is, $\hat{P}_{i}=K \tilde{P}_{i}$, for some constant $K>1$, for all $i$.

Proof: Since $\log D(\rho)$ is convex and increasing by Assumption $\Pi 1, D^{\prime}(\rho) / D(\rho)$ is nondecreasing in $\rho$. Note also that $D^{\prime}(\rho) / D(\rho)>0$ for $\rho \geq 0$, from the assumption $\Pi 1$. Thus, $D(\rho) / D^{\prime}(\rho)>0$ is nonincreasing in $\rho \geq 0$. From (22) and (25), respectively, follows that there exist unique $\tilde{\rho}$ and $\hat{\rho}$. Clearly, from (22) and (25), $\tilde{\rho}>\hat{\rho}$, and $\tilde{\rho}$, and thus $\tilde{\rho} / \hat{\rho}$, increases as $n$ increases. $\tilde{P}_{i}=\mu_{i}^{2} / D^{\prime}(\tilde{\rho})$ and $\hat{P}_{i}=\mu_{i}^{2} / D^{\prime}(\hat{\rho})$. Then, for all $i$,

$$
\hat{P}_{i}=K \tilde{P}_{i}, \quad K=\frac{D^{\prime}(\tilde{\rho})}{D^{\prime}(\hat{\rho})}>1 .
$$

d) Nash proportionate Pareto optimum flow control: We call the above-mentioned overall optimum the "Nash proportionate' Pareto optimum to the Nash equilibrium.

Consider the following measure of Pareto superiority [23]. Denote by $U_{i}^{a}(>0)$ the utility of user $i$ of an allocation $a$ of a system. Assume that the utilities of all users in question have a positive value. Consider that there are two allocations $a$ and $b$ corresponding to two different values of $\lambda$. Denote $\kappa_{i}=U_{i}^{a} / U_{i}^{b}$. If $\min _{i} \kappa_{i}>1$, we can say that $a$ is strongly Pareto superior to $b$. If $\min _{i} \kappa_{i}=1, a$ is Pareto indifferent or Pareto superior to $b$. If $\min _{i} \kappa_{i}<1, a$ is Pareto indifferent or Pareto inferior to $b$. Then, we use $\kappa=\min _{i} \kappa_{i}$ as a measure of strong Pareto superiority. Thus, $K$ defined above by (26) is regarded as the degree of Pareto superiority of the Pareto-optimal flow control over the Nash equilibrium.

From (22) and Lemma 2, as $n$ increases, $\tilde{\rho}$, and, thus, $D^{\prime}(\tilde{\rho})$ increases, while $\hat{\rho}$ and, thus, $D^{\prime}(\hat{\rho})$ remains the same, as seen from (25). Thus, $K$ increases as $n$ increases, which means the following.

Proposition 2: The degree of Pareto superiority of the Nash proportionate Pareto-optimal flow control over the Nash equilibrium of noncooperative flow control increases as the number of independent users increases.

2) A Special Case: Series-Parallel Channels: Consider the case where the network consists of $\sigma$ parallel paths each of which is composed of a series of $\chi$ identical links, that is, the network composes series-parallel queues. A random choice of a path is made by each user for each packet with an equal probability $1 / \sigma$ where choices are made independently of past choices.

$$
D(\rho)=\frac{\chi}{1-\rho / \sigma}
$$

Clearly, the $D(\rho)$ given by (27) satisfies the assumption $\Pi 1$. Then, from (27), $D / D^{\prime}=\sigma-\rho$. Then, for the noncooperative optimal flow control,

$$
\begin{aligned}
& \tilde{\rho}=\sigma n /(n+1), \tilde{\rho}_{i}=\sigma /(n+1) . \\
& \text { Therefore, } \tilde{P}_{i}=\mu_{i}^{2} \sigma /\left\{(n+1)^{2} \chi\right\} .
\end{aligned}
$$

For the Nash-proportionately-fair Pareto-optimal flow control,

$$
\hat{\rho}=\sigma / 2 \text {. Then, the optimum can be }
$$

achieved by $\hat{\rho}_{i}=\sigma /(2 n)$. Then $\hat{P}_{i}=\mu_{i}^{2} \sigma /(4 n \chi)$.

Thus, $K=\hat{P}_{i} / \tilde{P}_{i}=(n+1)^{2} /(4 n)$, and $K>1$ for $n \geq 2, K \rightarrow \infty$ $(n \rightarrow \infty)$. Note, in passing, that

$$
\tilde{\rho}_{i}=\frac{\sigma}{n+1}, \hat{\rho}_{i}=\frac{\sigma}{2 n}, \text { and, therefore, } \frac{\hat{\lambda}_{i}}{\tilde{\lambda}_{i}}=\frac{n+1}{2 n}<1,
$$

$D(\tilde{\rho})=\chi(n+1), D(\hat{\rho})=2 \chi$, and, therefore, $\frac{\hat{T}_{i}}{\tilde{T}_{i}}=\frac{2}{n+1}<1$.

Thus, in the Nash-proportionately-fair Pareto-optimal flow control, each user injects less flow and has better responsiveness than in the Nash equilibrium. Some numerical examples are as in the following. Recall that $n$ is the number of users.

For 9 users, in the Nash equilibrium, each user injects the rate of packets of 1.8 times, and receives the expected end-toend packet delay of 5 times and the power of 0.36 times as large as those in the Nash-proportionately-fair Pareto-optimal flow control.

For 99 users, in the Nash equilibrium, each user injects the rate of packets of 1.98 times, and receives the expected end-toend packet delay of 50 times and the power of 0.0396 times as 
large as those in the Nash-proportionately-fair Pareto-optimal flow control.

For 999 users, in the Nash equilibrium, each user injects the rate of packets of 1.998 times, and receives the expected endto-end packet delay of 500 times and the power of 0.003996 times as large as those in the Nash-proportionately-fair Paretooptimal flow control.

Remark 4: Thus, one of the contributions of the paper is showing the case where Nash equilibria become less efficient as the number of users increases. This is in contrast with previous results [11], [12] that we have obtained for routing games where we presented an example where the equilibrium is efficient for a large number of players and becomes inefficient as the number of players decrease. In that example, as the number of players tends to infinity, the equilibrium coincides with an overall optimal solution.

It should be noticed that the inefficiency results that we obatined for the flow control model hold for general topologies where as those that appear in routing games depend on the topology. For example, the original Braess paradox is known to occur for a specific 4-node topology, in contrast to the specific topologies of [11], [12].

Interestingly, our results on flow control apply directly to some routing games as well. Indeed, it is well known that flow control problems with additive costs, or even combined flow and routing control, can be transformed into equivalent routing control with no flow control [39] in which an extra link is added between each source-destination pair. This transformation can also be used for the Power criterion since maximizing the power is equivalent to maximize the log of the power which is additive.

\section{Noncooperative Flow Control with Additive Costs}

In this subsection, we briefly touch on another case of each user's objective. Consider the network described in Section III-A, and assume that the cost per packet over link $k$ is given by the function $\left(1 / \mu_{i k}\right) T^{(k)}\left(\rho_{k}\right)$ (given by (5)) where

$$
\rho_{k}=\sum_{r} \rho_{r k}, \quad \rho_{i k}=Q_{i k} \lambda_{i}
$$

The total cost paid by player $i$ is thus

$$
J_{i}(\lambda)=\lambda_{i} T_{i}=\sum_{l \in \boldsymbol{m}} \rho_{i l} T^{(l)}\left(\rho_{l}\right) .
$$

The utility for player $i$ is then given by

$$
U_{i}(\lambda)=R_{i}\left(\lambda_{i}\right)-a_{i} J_{i}(\lambda)
$$

where $R_{i}$ is positive and is increasing and concave in its argument and $a_{i}$ is a positive constant. Utilities with the above structure are common in telecommunication networks (see, for example, Alpcan and Başar [40], [41] that study special cases of such utilities).

Clearly, given the strategies, $\lambda^{(-i)}$, of other users, user $i$ optimizes $U_{i}$ by choosing its strategy $\check{\lambda}_{i}$, which is unique given $\lambda^{(-i)}$, and

$$
0 \leq \check{\lambda}_{i}<\min _{l}\left\{A_{i l} /\left(s_{l} Q_{i l}\right)\right\} .
$$

If we have $\tilde{\lambda}$ such that, given $\tilde{\lambda}^{(-i)}$ as $\lambda^{(-i)}, \check{\lambda}_{i}=\tilde{\lambda}_{i}$ holds for all $i, \tilde{\lambda}$ is a Nash equilibrium. A proof of the existence of a Nash equilibrium, $\tilde{\lambda} \in \boldsymbol{C}$, can be shown based on the Kakutani fixed-point theorem [38].

Theorem 4: There exists a Nash equilibrium flow control solution, $\tilde{\lambda} \in \boldsymbol{C}$, for this network.

Proof: See Appendix A.

Since $T^{(k)}$ is strictly increasing in its argument for all $k$, then $\Psi 1$ holds. Therefore, from Theorem 1 , it is seen that, if more than one user has the positive $\tilde{\lambda}_{i}$ in a Nash equilibrium, $\Psi 2$ holds as well, and it is strongly Pareto inefficient.

Corollary 2: A Nash equilibrium for flow control where each user optimizes its utility $U_{i}(\boldsymbol{\lambda})(28)$ is always strongly Pareto inefficient if Assumption $\Psi 2$ holds.

We note from (28) that $\left.\frac{d U_{i}}{d \lambda_{i}}\right|_{\lambda_{i}=0}=\left.\frac{d R_{i}}{d \lambda_{i}}\right|_{\lambda_{i}=0}-\left.a_{i} T_{i}(\lambda)\right|_{\lambda_{i}=0}$. Then, if $\left.\frac{d R_{i}}{d \lambda_{i}}\right|_{\lambda_{i}=0} \leq a_{i} T_{i}(\mathbf{0})$, clearly, $\tilde{\lambda}_{i}=0$. by noting the definition of $T_{i}$ and $R_{i}$. Therefore, the necessary condition that $\Psi 2$ holds is that $\left.\frac{d R_{i}}{d \lambda_{i}}\right|_{\lambda_{i}=0}>a_{i} T_{i}(\mathbf{0})$ holds for at least two $i$ 's.

\section{Concluding Remarks}

In this article, a general framework of strongly Paretoinefficient Nash equilibria in noncooperative games competing for common-pool resources has been presented. In particular, we have considered noncooperative flow control. We have firstly shown that the situation where each user optimizes its power unilaterally has a strongly Pareto-inefficient Nash equilibrium. For a subcategory of the networks of the situation, furthermore, we have obtained the explicit flow control solutions of the inefficient Nash equilibrium. We have also shown that, in some flow-control games, the degree of Pareto superiority of the Nash proportionate Pareto optimum to the Nash equilibrium in noncooperative flow control can increase as the number of users increases. We have then considered another utility of additive costs and have shown that an inefficient Nash equilibrium exists with respect to it.

In contrast, the general framework covers neither noncooperative network routing nor noncooperative load balancing in distributed systems. It may not be easy to extend the general framework to cover them with keeping its simplicity.

Appendix A. A Proof of the Existence of a Nash Equilibrium in Noncooperative Flow Control (Theorems 2 and 4)

In this appendix, we give a proof of the existence of a Nash equilibrium in noncooperative flow control as given in Subsections III-B and III-C. We consider the utility function $\hat{u}_{i}(\lambda)=\exp \left\{U_{i}\right\}=\exp \left\{R_{i}\left(\lambda_{i}\right)-a_{i} J_{i}(\lambda)\right\}$ for Subsection III-C. Then, both utility functions, $P_{i}(\lambda)$ for Subsection III-B and $\hat{u}_{i}(\lambda)$ for Subsection III-C, have non-negative finite values for $\lambda \in \boldsymbol{C}$ and the value zero for $\lambda \in \hat{\boldsymbol{C}}-\boldsymbol{C}$. In the following part 1 ), we first show that there exists a Nash equilibrium in region $\hat{\boldsymbol{C}}$ with the above utility functions. Then, in part 2), we show that such a Nash equilibrium is in region $\boldsymbol{C}$, which is a really feasible region considering the achievability of the statistical equilibrium of the networks considered. 
1) Consider the following function, $\phi_{i}$, for arbitrary $i$, whose domain is $\hat{\boldsymbol{C}}$, defined as follows. $\phi_{i}: \lambda \in \hat{\boldsymbol{C}} \rightarrow\left(\check{\lambda}_{i}, \lambda^{(-i)}\right) \in \hat{\boldsymbol{C}}$. That is, given $\lambda \in \hat{\boldsymbol{C}}$, the function $\phi_{i}$ gives the $\breve{\lambda}_{i}$ as follows with other $\lambda^{(-i)}$ being unchanged: $\check{\lambda}_{i}$ is uniquely given, if $A_{i l}>$ 0 for all $l$, by (15) for Subsection III-B and by the statement above (29) for Subsection III-C and $\breve{\lambda}_{i}=0$, if $A_{i l}=0$ for some $l$. Note that, for Subsection III-C, in the case where $A_{i l}>0$ for all $l, \breve{\lambda}_{i}$ is determined to be the same value, regardless of whether $\hat{u}_{i}$ or $U_{i}$ is used for the utility of user $i$. In the case where $A_{i l}=0$ for some $l, \breve{\lambda}_{i}$ is determined independently of the shape of the utility function of user $i$.

From (15) for Subsection III-B and from the statement above (29) for Subsection III-C, it is clearly seen that, for $\lambda \in \hat{\boldsymbol{C}}$, such that $A_{i l}>0, l \in \boldsymbol{k}, \phi_{i}$ is a continuous function of $\lambda \in \hat{\boldsymbol{C}}$ to $\left(\check{\lambda}_{i}, \boldsymbol{\lambda}^{(-i)}\right) \in \hat{\boldsymbol{C}}$. Furthermore, from (11) for Subsection III-B and (29) for Subsection III-C, as $A_{i l} \rightarrow 0$ for an arbitrary $l$ with $\lambda$ remaining in $\hat{\boldsymbol{C}}, \breve{\lambda}_{i} \rightarrow 0$. For such $\lambda$ that $A_{i l}=0$ for some $l, \breve{\lambda}_{i}$ keeps to be 0 while $\lambda$ remains in $\hat{\boldsymbol{C}}$. Therefore, $\phi_{i}$ is a continuous function of $\lambda$, for $\lambda \in \hat{\boldsymbol{C}}$ and $i \in \boldsymbol{n}$. Thus, $\phi_{i}$ is a continuous function of $\lambda \in \hat{\boldsymbol{C}}$ into $\hat{\boldsymbol{C}}$.

Consider a function $\phi=\phi_{1} \circ \phi_{2} \circ \cdots \circ \phi_{n}$. From the above, we see that $\phi$ is a continuous function and maps $\lambda \in \hat{\boldsymbol{C}}$ to $\breve{\lambda} \in \hat{\boldsymbol{C}}$. By noting that $\hat{\boldsymbol{C}}$ is a compact set, from the Kakutani fixed point theorem [38], the function $\phi$ has a fixed point $\tilde{\lambda}$ such that $\phi(\tilde{\lambda})=\tilde{\lambda}, \tilde{\lambda} \in \hat{\boldsymbol{C}}$. We can easily see that, if $\phi(\tilde{\lambda})=\tilde{\lambda}$, then $\phi_{1}(\tilde{\lambda})=\phi_{2}(\tilde{\lambda})=\cdots=\phi_{n}(\tilde{\lambda})=\tilde{\lambda}$. Thus, if $\phi(\tilde{\lambda})=\tilde{\lambda}$, $\tilde{\lambda}$ is a Nash equilibrium.

2) Clearly, $\lambda$, such that $1-s_{l} \sum_{r} Q_{r l} \lambda_{r}=0$ for some $l$, cannot be such a fixed point. Indeed, such $\lambda$ gives zero utilities $\left(P_{i}=0\right.$ and $\hat{u}_{i}=0$ for all $i$ ), and user $i$ such that $\lambda_{i}>0$ could increase its utility by decreasing its $\lambda_{i}$ (that is, if $\lambda_{i}>0$, then it must hold that $A_{i l}>0$ for all $l$. Then, from (11) for Subsection III-B and (29) for Subsection III-C, $\check{\lambda}_{i}$ mapped from $\lambda$ must be such that $1-s_{l} \sum_{r \neq i} Q_{r l} \lambda_{r}-s_{l} Q_{i l} \check{\lambda}_{i}=A_{i l}-s_{l} Q_{i l} \check{\lambda}_{i}>0$, and, thus, is less than $\lambda_{i}$ ). Therefore, a fixed point of $\phi, \tilde{\lambda}$, exists and $\tilde{\boldsymbol{\lambda}} \in \boldsymbol{C}$, which is a Nash equilibrium of the noncooperative flow control.

\section{REFERENCES}

[1] P. Dubey, "Inefficiency of Nash equilibria," Mathematics of Operations Research, vol. 11, pp. 1-8, 1986.

[2] D. Braess, "Über ein Paradoxen aus der Verkehrsplanung," Unternehmensforschung, vol. 12, pp. 258-268, 1968.

[3] J. D. Murchland, "Braess's paradox of traffic flow," Transpn. Res., vol. 4, pp. 391-394, 1970.

[4] M. Frank, "The Braess paradox," Mathematical Programming, vol. 20, pp. 283-302, 1981.

[5] J. E. Cohen and F. P. Kelly, "A paradox of congestion in a queuing network," J. Appl. Prob., vol. 27, pp. 730-734, 1990.

[6] F. P. Kelly, "Network routing," Phil. Trans. R. Soc. Lond. A, vol. 337, pp. 343-367, 1991 .

[7] J. E. Cohen and C. Jeffries, "Congestion resulting from increased capacity in single-server queueing networks," IEEE/ACM Trans. Networking, vol. 5, no. 2, pp. 305-310, April 1997

[8] Y. A. Korilis, A. A. Lazar, and A. Orda, "Architecting noncooperative networks," IEEE J. Selected Areas in Communications, vol. 13, pp. 1241-1251, 1995.

[9] Y. A. Korilis, A. A. Lazar, and A. Orda, "Avoiding the Braess paradox in noncooperative networks," J. Appl. Prob., vol. 36, pp. 211-222, 1999.

[10] T. Roughgarden and É. Tardos, "How bad is selfish routing?", J. ACM, vol. 49, no. 2, pp. 236-259, 2002.

[11] H. Kameda, E. Altman, T. Kozawa, and Y. Hosokawa, "Braess-like paradoxes in distributed computer systems," IEEE Trans. Automatic Control, vol. 45, no. 9, pp. 1687-1691, 2000.
[12] H. Kameda and O. Pourtallier, "Paradoxes in distributed decisions on optimal load balancing for networks of homogeneous computers," $J$. $A C M$, vol. 49, no. 3, pp. 407-433, 2002.

[13] D. Famolari, N. B. Mandayam, D. Goodman, and V. Shah, "A new framework for power control in wireless data networks: Games, utility and pricing," in Wireless Multimedia Network Technologies, Ganesh, Pahlavan, and Zvonar, Eds., pp. 289-310. Kluwer Academic Publishers, Jan. 1999.

[14] A. B. MacKenzie and S. B. Wicker, "Game theory and the design of self-configuring, adaptive wireless networks," IEEE Communications Magazine, vol. 39, no. 11, pp. 126-131, Nov. 2001.

[15] H. Ji and C-Y. Huang, "Non-cooperative uplink power control in cellular radio systems," Wireless Networks, vol. 4, pp. 233-240, 1998.

[16] G. Hardin, "The tragedy of the commons," Science, vol. 162, pp. 1243$1248,1968$.

[17] J. E. Roemer, "A public ownership resolutions of the tragedy of the commons," Social Philosophy E Policy, vol. 6, no. 2, pp. 74-92, 1989.

[18] J. E. Roemer and J. Silvester, "The proportional solution for economies with both private and public ownership," J. Economic Theory, vol. 59, pp. 426-444, 1993.

[19] Y. Funaki and T. Yamamoto, "The core of an economy with a common pool resource: A partition function form appraoch," Int. J. Game Theory, vol. 28, pp. 157-171, 1999.

[20] A. Giessler, J. Haanle, A. Konig, and E. Pade, "Free buffer allocation An investigation by simulation," Comput. Networks, vol. 1, pp. 191-204, 1978.

[21] Y. A. Korilis and A. A. Lazar, "On the existence of equilibria in noncooperative optimal flow control," J. ACM, vol. 42, no. 3, pp. 584613, May 1995.

[22] R. Mazumdar, L. G. Mason, and C. Doulgligeris, "Fairness in network optimal flow control: Optimality of product forms," IEEE Trans. Communications, vol. 39, no. 5, pp. 775-782, May 1991.

[23] H. Kameda, "Bounds on benefits and harms of adding connections to noncooperative networks," in NETWORKING 2004 - Proc. 3rd International IFIP-TC6 Networking Conference, Athens, Greece, May 9-14, 2004, Series: Lecture Notes in Computer Science, Vol. 3042 (N. Mitrou, et al., Eds.), Berlin, Germany, 2004, pp. 405-417, SpringerVerlag.

[24] T. Alpcan, T. Başar, R. Srikant, and E. Altman, "CDMA uplink power control as a noncooperative game," Wireless Networks, vol. 8, no. 6, pp. 659-670, 2002

[25] C. U. Saraydar, N. B. Mandayam, and D. J. Goodman, "Pricing and power control in a multicell wireless data network," IEEE JSAC, vol. 19, no. 10, pp. 1883-1892, Oct. 2001.

[26] C. U. Saraydar, N. B. Mandayam, and D. J. Goodman, "Efficient power control via pricing in wireless data networks," IEEE Trans. Communications, vol. 50, no. 2, pp. 291-303, Feb. 2002.

[27] F. Baskett, K. Mani Chandy, R. Muntz, and F. G. Palacios, "Open, closed, and mixed networks of queues with different classes of customers," J. ACM, vol. 22, no. 2, pp. 248-260, 1975.

[28] F. P. Kelly, "Mathematical modelling of the internet," in Bjorn Engquist and Wilfried Schmid (Eds.), Mathematics Unlimited - 2001 and Beyond. Springer, 2001.

[29] L. Kleinrock, "On flow control in computer networks," in Proc. IEEE ICC '78, 1978, pp. 27.2.1-27.2.5.

[30] L. Kleinrock, "Power and deterministic rules of thumb for probabilistic problems in computer communications," in Proc. IEEE Int. Conf. Commun. (ICC '79), 1979, pp. 43.1.1-43.1.10.

[31] C. Douligeris and R. Mazumdar, "A game theoretic perspective to flow control in telecommunication networks," J. Franklin Inst., vol. 329, pp. 383-402, 1992.

[32] M. Gerla and L. Kleinrock, "Flow control: A comparative survey," IEEE Trans. Communications, vol. 28, no. 4, pp. 553-574, April 1980.

[33] J. M. Jaffe, "Bottleneck flow control," IEEE Trans. Communications, vol. 29, no. 2, pp. 954-962, July 1981.

[34] D. Mitra, "Asymptotically optimal design of congestion control for high speed data networks," IEEE Trans. Communications, vol. 40, no. 2, pp. 301-311, May 1992.

[35] Z. Zhang and C. Douligeris, "Convergence of synchronous and asynchronous greedy algorithms in a multiclass telecommunications environment," IEEE Trans. Communications, vol. 40, no. 8, pp. 12771281, 1992.

[36] S. Stidham, Jr., "Optimal control of admission to a queueing system," IEEE Trans. Automatic Control., vol. 30, no. 8, pp. 705-713, Aug. 1985.

[37] H. Yaïche, R. R. Mazumdar, and C. Rosenberg, "A game theoretic framework for bandwidth allocation and pricing in broadband networks," IEEE/ACM Trans. Networking, vol. 8, no. 5, pp. 667-677, 2000. 
[38] S. Kakutani, "A generalization of Brouwer's fixed-point theorem," Duke Mathematical Journal, vol. 8, pp. 451-459, 1941.

[39] A. Haurie and P. Marcotte, "On the relationship between Nash-Cournot and Wardrop equilibria," Networks, vol. 15, pp. 295-308, 1985.

[40] T. Alpcan and T. Başar, "A game-theoretic framework for congestion control in general topology networks," in Proc. 41th IEEE CDC, Las Vegas, Nevada, Dec. 10-13 2002, (Available at http://decision.csl.uiuc.edu/alpcan/publications.htm).

[41] T. Alpcan and T. Başar, "A utility-based congestion control scheme for Internet-style networks with delay," in Proc. IEEE Infocom, 2003, (Available at http://decision.csl.uiuc.edu/ $/$ alpcan/publications.htm).

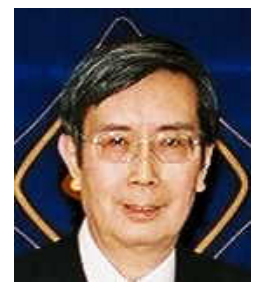

Hisao Kameda (M'99-SM'03-F'08) received the B.Sc. degree in physics and the M.Sc. and D.Sc. degrees for his work in computer science, all from the University of Tokyo, Japan. Since then, he has worked at University of Tokyo, IBM T.J. Watson Research Center, Yorktown Heights, NY, University of Toronto, ON, Canada, and the University of Electro-Communications, Chofu, Tokyo, Japan, among others. In 1992, he became a Professor at the Institute of Information Sciences and Electronics, University of Tsukuba, Japan. Since 2006, He has been a Professor Emeritus of the University of Tsukuba. He has been conducting research on operating system design principles, operating system scheduling, performance measurement and analysis of computer systems, and distributed and parallel processing, etc. He has also been continuously interested in general systems implications of computer systems. He is a Fellow of IEEE, IEICE, IPSJ, and ORSJ.

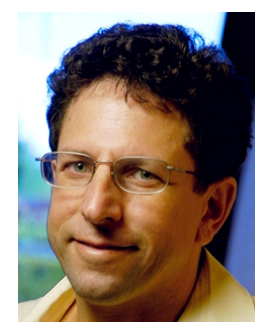

Eitan Altman (M'93-SM'00) received the B.Sc. degree in electrical engineering (1984), the B.A. degree in physics (1984) and the Ph.D. degree in electrical engineering (1990), all from the Technion-Israel Institute, Haifa. In (1990) he further received his B.Mus. degree in music composition in Tel-Aviv university. Since 1990, he has been with INRIA (National research institute in informatics and control) in Sophia-Antipolis, France. His current research interests include performance evaluation and control of telecommunication networks and in particular congestion control, wireless communications and networking games. He is in the editorial board of the scientific journals: WINET, JDEDs and JEDC, and served in the journals Stochastic Models, COMNET, SIAM SICON. He has been the general chairman and the (co)chairman of the program committee of several international conferences and workshops (on game theory, networking games and mobile networks). $\mathrm{He}$ has published more than 140 papers in international refereed scientific journals. More informaion can be found at http://www.inria.fr/mistral/personnel/Eitan.Altman/me.html 